\title{
Estimativa da evapotranspiração e coeficiente de cultivo da Heliconia bihai L., cultivada em ambiente protegido ${ }^{(1)}$
}

\author{
RUBENS SONSOL GONDIM (2), ANTÔNIA RENATA MONTEIRO GOMES ${ }^{(3)}$, \\ FRED CARVALHO BEZERRA ${ }^{(4)}$, CARLOS ALEXANDRE GOMES COSTA ${ }^{(5)}$ e NATANAEL SANTIAGO PEREIRA $^{(5)}$
}

\section{RESUMO}

O trabalho foi desenvolvido em ambiente protegido, do tipo telado, no Campo Experimental do Curu, região litorânea do Estado do Ceará, e teve como objetivo estimar a evapotranspiração e os coeficientes de cultivo da Heliconia bihai L. empregando o método do balanço hídrico e a evapotranspiração de referência (ETo) estimada pelo método de Penman-Monteith-FAO e tanque Classe A. Utilizou-se na cultura o espaçamento de 2,00 m entre fileiras por $1,00 \mathrm{~m}$ entre plantas e irrigação por microaspersão. A taxa média de ETc foi de $2,3 \mathrm{~mm} \mathrm{dia}{ }^{-1}$, totalizando $174,7 \mathrm{~mm}$ para todo o período. Com relação às fases fenológicas da cultura, observou-se uma ETc $2,2 \mathrm{~mm} \mathrm{dia}^{-1}$ durante a fase F2 (10\% até $80 \%$ de cobertura vegetal); $2,3 \mathrm{~mm} \mathrm{dia}^{-1}$ na fase $\mathrm{F} 3$ ( $80 \%$ de cobertura até o inicio da floração) e 2,5 $\mathrm{mm} \mathrm{dia}^{-1}$ na fase F4 (início da floração até a colheita). Os valores de coeficientes de cultivo (Kc) foram maiores quando a ETo foi estimada pelo tanque Classe A, em relação aos valores da ETo estimados pelo método de Penman-Monteith-FAO. Empregando o primeiro método, os valores de Kc foram 0,80; 1,20 e 1,20, enquanto para Penman-Monteith-FAO foram 0,$60 ; 0,80$ e 0,80 , nas fases F2; F3 e F4, respectivamente.

Palavras-chave: irrigação, flores tropicais.

\section{ABSTRACT}

Evapotranspiration and crop coefficient estimation for a greenhouse garden of Heliconia bihai L.

This task was developed in screen greenhouse conditions at Curu-Paraipaba Experimental Station, coast region of state of Ceará, Brazil and had as objective to estimate the evapotranspiration and crop coefficient for Heliconia bihai L using the soil water balance method. The crop coefficient was estimated from either Penman-Monteith and Class A pan evaporation ETo. The space used was $2.00 \mathrm{~m}$ between rows and $1.00 \mathrm{~m}$ between plants and they were microsprinkler irrigated. The average daily evapotranspiration observed was $2.3 \mathrm{~mm} \mathrm{day}^{-1}$, totalizing $174.7 \mathrm{~mm}$ for the whole cycle. Regarding to the crop phase it was $2.2 \mathrm{~mm}$ day $^{-1}$ for F2 phase ( $10 \%$ to $80 \%$ crop coverage), 2.3 $\mathrm{mm}$ day $^{-1}$ for $\mathrm{F} 3$ phase ( $80 \%$ of crop coverage to blossom beginning) and $2.5 \mathrm{~mm} \mathrm{day}^{-1}$ for $\mathrm{F} 4$ phase (blossom beginning to harvest). The crop coefficients were higher when estimated by Class A pan evaporation ETo, when comparing to those estimated by Penman-Monteith-FAO method. The results for F2 (beginning of water balance to $80 \%$ crop coverage), F3 (80\% crop coverage until blossom beginning) and F4(from blossom beginning to harvest) were $0,80,1,20$ e 1,20 respectively, for Class A pan evaporation and 0,60, 0,80 e 0,80 for Penman-Monteith-FAO.

Key words: irrigation, tropical flowers

\section{INTRODUÇÃO}

A floricultura está inserida na agricultura irrigada e por apresentar vantagens tais como: maior rentabilidade por área cultivada, retorno mais rápido dos investimentos aplicados e capacidade de geração de empregos, pode ser vista como uma alternativa atrativa ao agricultor brasileiro.

O Brasil apresenta potencial para tornar-se um grande produtor e exportador de flores e plantas ornamentais, destacando-se as tropicais que por apresentar cores intensas e maior durabilidade póscolheita, vem despertando, a cada ano, maior interesse por parte dos consumidores.
Merece destaque a produção de helicônias, que é uma flor tropical bastante cultivada no momento. São plantas rizomatosas, herbáceas, cujas inflorescências são protegidas por brácteas que apresentam uma variedade de cores e formatos. Por ser uma flor de origem tropical adaptase muito bem a nossa região, desde que sejam adotadas tecnologias específicas.

Dispõe-se de poucas informações sobre o comportamento das helicônias em condições de irrigação. BEZERRA (1997) cita que o suprimento de água de irrigação de boa qualidade deve ser garantido durante todo o ciclo da cultura. CASTRO (1995) sugere que sejam feitas irrigações em abundância, principalmente após a emissão das folhas, mantendo o teor de umidade do solo elevado.

\footnotetext{
${ }^{1}$ Recebido em 16/03/2006 e aprovado para publicação em 23/07/2007

${ }^{2}$ Eng ${ }^{\circ}$. Agrônomo, Pesquisador da Embrapa Agroindústria Tropical. rubens@cnpat.embrapa.br. Aluno de Doutorado no Departamento de Engenharia Hidráulica e Ambiental da UFC.

Eng. Agrônoma, M.Sc. Irrigação e Drenagem. Bolsista do CNPq

${ }^{4}$ Eng ${ }^{\circ}$. Agrônomo. Doutor em Fisiologia Vegetal. Pesquisador da Embrapa Agroindústria Tropical.

Estudantes de Agronomia. Estagiários na Embrapa Agroindústria Tropical.
} 
Em locais secos, são recomendadas de duas a três irrigações por semana, evitando-se, contudo a saturação do solo. O sistema de irrigação mais recomendado é a microaspersão e segundo LAMAS (2001), a helicônia é sensível à falta de umidade no solo, podendo afetar a qualidade do produto. Todavia essas informações não fornecem dados precisos que possam possibilitar uma programação racional das irrigações, havendo, portanto, necessidade de serem realizadas pesquisas para melhor definir informações acerca das necessidades hídricas da helicônia que possam promover o aumento da produtividade e qualidade das flores.

Com esse intuito, o presente trabalho objetivou estimar a evapotranspiração e os coeficientes de cultivo da Heliconia bihai L.

\section{MATERIALEMÉTODOS}

O experimento foi conduzido no Campo Experimental do Curu, pertencente à Embrapa Agroindústria Tropical, latitude $3^{\circ} 28^{\prime} 47^{\prime}$ ' S, longitude 39॰09' 47" W e altitude de $31 \mathrm{~m}$, localizado no município de Paraipaba-CE. O clima da região segundo Köppen, é do tipo Aw', classificado como tropical chuvoso e caracterizado por apresentar o máximo de chuvas no outono e período seco no inverno. A precipitação média anual do município de Paraipaba é de 986 mm, com evaporação de $2.625 \mathrm{~mm}$, umidade relativa média de $86 \%$ e temperatura média anual de $27,1^{\circ} \mathrm{C}$. O solo da área experimental classifica-se como Neossolo Quartzarênico.

Os dados climáticos referentes ao período de condução do experimento, observados na Estação agrometeorológica convencional, distante $500 \mathrm{~m}$ da área experimental, e na mini-estação instalada dentro da área experimental, são apresentados nas tabelas 1 e 2 respectivamente.

A cultura estudada foi a Helicônia (H. bihai L.), cultivada em ambiente protegido totalmente coberto com malha de sombreamento (50\%), plantada em 03/10/2003, no espaçamento de 2,00 $\mathrm{m}$ entre fileiras por $1,00 \mathrm{~m}$ entre plantas, numa área experimental de $540 \mathrm{~m}^{2}$ Foram feitas adubações orgânicas e minerais a cada três meses, utilizando-se húmus de minhoca $\left(5 \mathrm{Kg} \mathrm{m}^{-2}\right)$ e $62,5 \mathrm{~g} \mathrm{cova}^{-1}$ da fórmula NPK 15-15-15 e FTE-BR 12 (3,75 Kg ha'-1).

O sistema de irrigação utilizado foi do tipo microaspersão, cujos emissores apresentavam uma vazão de $74 \mathrm{~L} \mathrm{~h}^{-1}$. O controle das irrigações foi realizado através de tensiômetros instalados a $0,10 \mathrm{~m} \mathrm{e} 0,30 \mathrm{~m}$ sendo que os instalados a $0,10 \mathrm{~m}$ serviram para indicar o momento da irrigação (tensão de $10 \mathrm{kPa}$ ) e juntamente com as leituras do tensiômetro a 0,30 m, o cálculo da lâmina de irrigação.

A evapotranspiração da cultura (ETc) foi estimada utilizando-se o método do balanço hídrico do solo, realizado de acordo com a metodologia apresentada por Reichardt (1985), (Equação 1), para a profundidade de 0 a 0,6 m. Foram utilizados dados de três baterias de tensiômetros de mercúrio, instalados nas profundidades de $0,10,0,30,0,50$ e 0,70 m. O balanço hídrico foi realizado no período de 01/12/2003, 60 dias após o plantio das mudas, até $08 / 04 / 2004$, início da colheita.

$$
\mathrm{ETc}=\mathrm{P}+\mathrm{I} \pm \mathrm{Q}_{\mathrm{z}}-\Delta A
$$

em que:

ETc - evapotranspiração da cultura (mm)

$\mathrm{P}$ - precipitação pluvial (mm)

I - irrigação (mm)

$\mathrm{Q}_{\mathrm{z}}$, - , drenagem profunda ou ascensão capilar (mm)

$\Delta A$ - variação do armazenamento da água do solo na camada de profundidade de 0 a $0,60 \mathrm{~m}$ para o intervalo de tempo considerado no balanço.

A drenagem profunda e a ascensão capilar para a profundidade de $0,60 \mathrm{~m}$, foram determinadas utilizandose a equação de Buckingham - Darcy, escrita de uma maneira simplificada por REICHARDT (1985), da seguinte forma:

$$
\mathrm{QZ}=-\mathrm{K}(\theta) \frac{\Delta \Psi t}{\Delta Z}
$$

em que,

Qz - fluxo de água ( $\left.\mathrm{cm} \mathrm{dia}^{-1}\right)$;

$\mathrm{K} .(\theta)$ - condutividade hidráulica do solo, em função da umidade do solo $\left(\mathrm{cm} \mathrm{dia}^{-1}\right)$;

$\frac{\Delta \Psi t}{\Delta Z}$ - gradiente do potencial total da água no solo $\left(\mathrm{cm} \mathrm{cm}^{-1}\right)$.

Aplicando-se a Equação 2 para a direção vertical, na profundidade máxima de controle do solo, $\mathrm{Z}=0,60 \mathrm{~m}$, obtém-se a seguinte equação:

$$
Q z=-K(\theta)_{0,60}\left\{\frac{\Psi_{\mathrm{T}}^{\prime} 50-\Psi_{\mathrm{T}} 70}{20}\right\}_{0,60}
$$

em que,

$\left\{\frac{\Psi_{\mathrm{T}} 50-\Psi_{\mathrm{T}}^{\prime} 70}{20}\right\}_{0,60}$

- gradiente do potencial total da água no solo, obtido a partir dos potenciais totais a $0,50 \mathrm{~m}$ e $0,70 \mathrm{~m}$ $\mathrm{H}_{2} \mathrm{O}$;

Os valores de $\mathrm{K}(\theta)_{0,60}$, em $\mathrm{mm} \mathrm{dia}{ }^{-1}$, foram obtidos mediante ensaio de campo conduzido na área experimental, ajustando-se a uma equação, conforme metodologia sugerida por de HILLEL et al. (1972):

$$
\mathrm{K}(\theta)_{0,60=} 2^{10 \mathrm{e}(87,677(\theta-0,353))}
$$

A determinação da variação do armazenamento da água no solo na profundidade e no intervalo de tempo considerado foi obtida mediante a expressão de REICHARDT(1985):

$$
\Delta A=\left(\bar{\theta}_{2}-\bar{\theta}_{1}\right) Z
$$

em que,

$\triangle A$ - variação do armazenamento da água do solo; $\overline{\theta_{2}}$ - umidade média até a profundidade de $0,60 \mathrm{~m}$, no dia da irrigação $\left(\mathrm{cm}^{3} \mathrm{~cm}^{-3}\right)$; 
$\bar{\theta}_{1}$ - umidade média até a profundidade de $0,60 \mathrm{~m}$, no dia da irrigação anterior $\left(\mathrm{cm}^{3} \mathrm{~cm}^{-3}\right)$;

$\mathrm{Z}$ - profundidade adotada para o balanço $(0,60 \mathrm{~m})$.

Em função da área em estudo apresentar declividade mínima e as irrigações terem sido controladas de modo a não permitir a ocorrência de escoamento superficial, este foi considerado nulo.

Para determinação da evapotranspiração de referência (ETo) foram utilizados os métodos de PenmanMonteith-FAO (ALLEN et al., 1998) e do tanque Classe A. Para o cálculo da ETo pelo método de Penman-MonteithFAO, utilizaram-se dados meteorológicos obtidos em uma estação agrometeorológica distante $500 \mathrm{~m}$ da área experimental. Com relação ao método do tanque Classe A, utilizaram-se dados obtidos de um tanque Classe A instalado dentro do ambiente protegido.

Os coeficientes de cultivo $(\mathrm{Kc})$ foram calculados segundo a equação:

$$
\mathrm{Kc}=\mathrm{ETc} / \mathrm{ETo}
$$

em que,

ETc - evapotranspiração da cultura $\left(\mathrm{mm} \mathrm{dia}^{-1}\right)$;

ETo - evapotranspiração de referência $\left(\mathrm{mm} \mathrm{dia}^{-1}\right)$.

De acordo com as observações de caráter morfológico, o desenvolvimento da cultura foi dividido em quatro fases fenológicas: F1 (do plantio até 10\% de cobertura vegetal), F2 (10\% até $80 \%$ de cobertura vegetal), F3 ( $80 \%$ de cobertura até o inicio da floração) e F4 (início da floração até a colheita).

O monitoramento da cobertura vegetal foi realizado semanalmente com auxilio de fotografias obtidas verticalmente a uma altura de aproximadamente $2 \mathrm{~m}$. No processamento das imagens digitais utilizou-se um programa desenvolvido pela EMBRAPA(SIARC - Sistema Integrado para Análise de Raízes e Cobertura do Solo).

\section{RESULTADOS E DISCUSSÃO}

Na figura 1 pode-se observar a variação da evapotranspiração de referência (ETo) determinada pelos métodos do tanque Classe A e Penman-Monteith-FAO, e evapotranspiração da cultura (ETc) determinada pelo balanço hídrico, em função do número de dias após o plantio (DAP). Observa-se que a ETo calculada pelo método de Penman-Monteith-FAO foi maior que a ETo calculada pelo método do tanque Classe $\mathrm{A}$, apresentando um valor médio de 3,76 mmdia ${ }^{-1}$ e 2,56 $\mathrm{mm} \mathrm{dia}^{-1}$, respectivamente, durante todo o período estudado.

Conforme observado por OMETO (1981) e BASTOS (1994), o tanque Classe A normalmente superestima os valores da ETo, quando comparado a Penman-Monteith, em função de sua geometria e do pequeno volume de água que comporta. Resultados semelhantes foram observados por VALIATI et al., (2003) que concluíram que o tanque Classe A superestima em média, $4,12 \%$ a evapotranspiração em ambiente protegido, em relação ao método de Penman-Monteith.
Entretanto, em função da pequena diferença encontrada, entre os dois métodos, o mesmo autor recomenda o uso do tanque Classe A para estimativa da evapotranspiração em ambiente protegido.

No presente trabalho ocorreu o oposto, provavelmente em razão do tanque Classe A encontrar-se em ambiente protegido (com sombreamento) e na escolha do coeficiente do tanque (Kp) foram consideradas as condições meteorológicas (umidade relativa do ar e velocidade do vento) medidas, portanto, no interior do ambiente protegido, que condicionaram valores de evapotranspiração menores em relação aos obtidos pelo método de Penman-Monteith-FAO, este, estimado com dados do ambiente externo.

A taxa média de ETc foi de $2,3 \mathrm{~mm} \mathrm{dia}^{-1}$, totalizando $174,7 \mathrm{~mm}$ para todo o período. Com relação às fases fenológicas da cultura, observou-se uma ETc 2,2 mm dia $^{-1}$ durante a fase F2 (10\% até $80 \%$ de cobertura vegetal); $2,3 \mathrm{~mm} \mathrm{dia}^{-1}$ na fase $\mathrm{F} 3(80 \%$ de cobertura até o inicio da floração) e 2,5 mm dia-1 na fase F4 (início da floração até a colheita).

$\mathrm{Na}$ tabela 3 são apresentados os coeficientes de cultivo (Kc) médios para cada fase fenológica (F). Não foram calculados valores de coeficiente de cultivo $(\mathrm{Kc})$ referentes à fase inicial, pois o balanço hídrico iniciou-se aos 60 dias após o plantio.

Os valores do coeficiente de cultivo (Kc) obtidos através da evapotranspiração de referência estimados pelo tanque Classe A instalado dentro do ambiente protegido revelaram-se maiores do que aqueles obtidos pelo Penman-Monteith-FAO, conforme pode ser visto na tabela 3 . Dessa forma, os valores médios dos coeficientes de cultivos encontrados para as fases F2, F3 e F4 foram 0,$80 ; 1,20$ e 1,20 respectivamente, quando utilizou-se o método do tanque Classe A, e 0,60; 0,80 e 0,80 para o método de Penman-Monteith-FAO.

SCATOLINI (1996) estudando modelos para estimativa da evapotranspiração do interior de ambientes protegidos chegou à conclusão que o ambiente protegido altera os elementos meteorológicos de maneira não uniforme, dificultando a estimativa da evapotranspiração a partir de elementos externos. Portanto, o coeficiente de cultivo determinado a partir do tanque Classe A pode ser recomendado para o manejo de irrigação da helicônia, uma vez que, o tanque Classe A utilizado na determinação da evapotranspiração de referência encontrava-se submetido às mesmas condições de sombreamento e climáticas da cultura.

\section{CONCLUSÕES}

A helicônia apresentou uma taxa de evapotranspiração média de $2,2 \mathrm{~mm} \mathrm{dia}^{-1}$ durante a fase F2 ( $10 \%$ até $80 \%$ de cobertura vegetal); $2,3 \mathrm{~mm} \mathrm{dia}^{-1}$ na fase $\mathrm{F} 3$ ( $80 \%$ de cobertura até o inicio da floração) e 2,5 $\mathrm{mm} \mathrm{dia}^{-1}$ na fase F4 (início da floração até a colheita)

Os valores de ETo apresentaram variação em função do método utilizado. Os valores estimados pelo 
tanque Classe A mantiveram-se inferiores aqueles estimados pelo método de Penman-Monteith-FAO. Consequentemente, os valores de Kc obtidos por meio da ETo estimada no tanque, revelaram-se maiores do que aqueles utilizando-se da ETo estimada pelo método de Penman-Monteith-FAO. Dessa forma os valores médios dos coeficientes de cultivos encontrados para as fases F2, F3 e F4 foram 0,80; 1,20 e 1,20 respectivamente, quando utilizou-se o método do tanque Classe A, e 0,60; 0,80 e 0,80 para o método de Penman-Monteith-FAO.

\section{REFERÊNCIAS}

ALLEN, R.G.; PEREIRA, L.S.; RAES, D.; SMITH, M. Crop evapotranspiration: guidelines for computing crop water requirements. Rome: FAO, 1998. 300p. (FAO. Irrigation and Drainage Paper, 56).

BASTOS, E. A Determinação dos coeficientes de cultura da alface (Lactuca sativa L.). Botucatu, 1994. 101f. Dissertação (Mestrado em Irrigação e Drenagem)Faculdade de Ciências Agronômicas, Universidade Estadual Paulista.

BEZERRA, F. C. Curso de floricultura: aspectos gerais e técnicas de cultivo para flores tropicais. Fortaleza: EMBRAPA-CNPAT, 1997.38p.
CASTRO, C. E. F. de. Helicônia para exportação: aspectos técnicos da produção. Brasília: Embrapa / SPI, 1995. 44p. (Série Publicações Técnicas Frupex; 16).

HILLEL, D.; KRENTOS, V D.; STYLIANOU, Y. Procedure and test of na internal drainage method for measuring soil hydraulic characteristics in situ. Soil Science, Baltimore, v.114, p: 395 - 400, 1972.

LAMAS, A. M. Floricultura tropical: técnicas de cultivo. Recife. SEBRAE/PE, 2001.(Série Emprendedor 5).

OMETTO, J.C. Bioclimatologia vegetal. São Paulo: ed. Agronômica Ceres, 1981.425p.

REICHARDT, K. Processo de transferências no sistema solo-planta-atmosfera. 4. ed. Campinas; Fundação Cargill, 1985.466p.

SCATOLINI, M. E. Estimativa da evapotranspiração da cultura de crisântemo em estufa a partir de elementos meteorológicos. 1996. 71f. Dissertação (Mestrado em Agronomia - Irrigação e Drenagem) - Escola Superior de Agricultura "Luiz de Queiroz”, Piracicaba.

VALIATI, M. I; DUTRA, I.; CUNHA, A.R. da.; ESCOBEDO, J.F. Estimativa da evapotranspiração potencial em ambiente protegido utilizando o método do tanque Classe A e de Penman-Monteith. In: CONGRESSO BRASILEIRO DE AGROMETEOROLOGIA, 13, 2003, Piracicaba-SP. Anais... Piracicaba: Sociedade Brasileira de Agrometeorologia. 2003, p. 63-64.

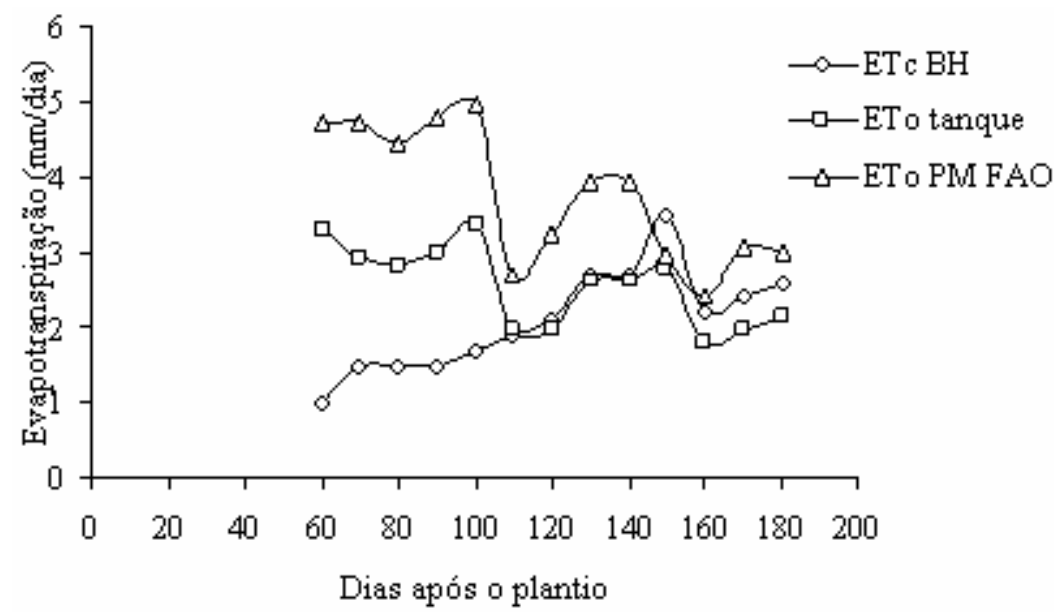

Figura 1. Comportamento da evapotranspiração da helicônia obtida pelo método do balanço hídrico e da evapotranspiração de referência obtida pelos métodos do tanque Classe A em ambiente protegido e Penman-MonteithFAO.

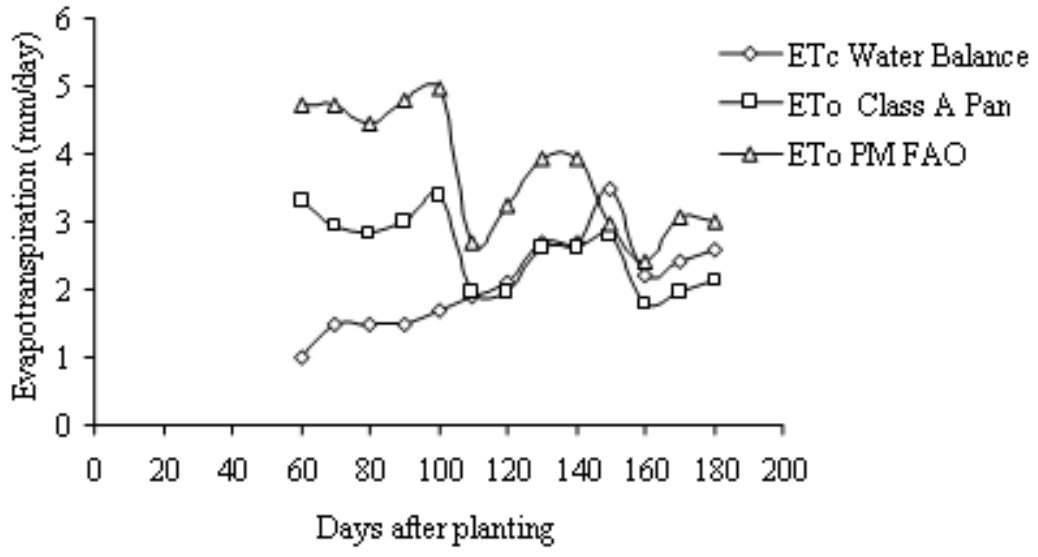

Figure 1. Heliconia evapotranspiration from water balance approach and reference evapotranspiration from Class A pan evaporation inside greenhouse and Penman-Monteith-FAO from weather station. 
Tabela 1. Médias mensais de temperatura, umidade relativa, velocidade do vento, radiação e evapotranspiração de referência e totais mensais da precipitação, obtidos na estação agrometeorológica convencional. Paraipaba, CE, 2003-2004.

Table 1. Average monthly temperature, relative air humidity, wind speed, radiation, reference evapotranspiration and total monthly precipitation from conventional agrometeorological station,. Paraipaba, CE, 2003-2004.

\begin{tabular}{lcccccc}
\hline Months & $\begin{array}{c}\text { Air Temperature } \\
\left({ }^{\circ} \mathrm{C}\right)\end{array}$ & $\begin{array}{c}\text { Relative } \\
\text { humidity }(\%)\end{array}$ & $\begin{array}{c}\text { Wind sp } \\
\left(\mathrm{m} \mathrm{s}^{-2}\right)\end{array}$ & $\begin{array}{c}\text { Radiation } \\
\left(\mathrm{MJ} \mathrm{m}^{-2}\right)\end{array}$ & $\begin{array}{c}\text { ETo } \\
(\mathrm{mm})\end{array}$ & $\begin{array}{c}\text { Precipitation } \\
(\mathrm{mm})\end{array}$ \\
\hline Dec & 29,0 & 63 & 2,3 & 21,2 & 4,6 & 5,0 \\
Jan & 27,9 & 78 & 1,4 & 15,7 & 3,4 & 388,2 \\
Feb & 27,6 & 79 & 1,1 & 16,1 & 3,3 & 179,3 \\
Mar & 23,4 & 79 & 1,2 & 16,5 & 3,4 & 290,3 \\
Apr & 25,3 & 83 & 1,2 & 16,1 & 3,2 & 104,6 \\
\hline
\end{tabular}

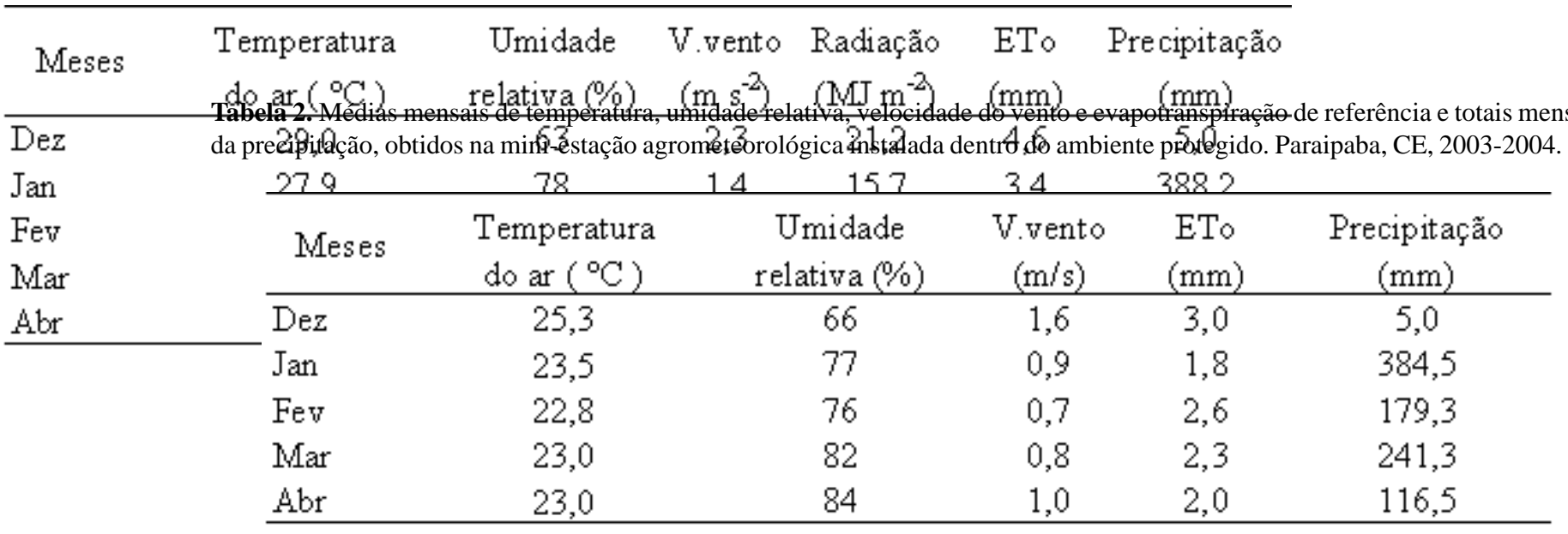

Table 2. Average monthly temperature, relative air humidity, wind speed, radiation, reference evapotranspiration and total monthly precipitation from inside greenhouse mini station, Paraipaba, CE, 2003-2004.

\begin{tabular}{cccccc}
\hline Months & $\begin{array}{c}\text { Air Temperature } \\
\left({ }^{\circ} \mathrm{C}\right)\end{array}$ & $\begin{array}{c}\text { Relative air } \\
\text { humidity }(\%)\end{array}$ & $\begin{array}{c}\text { Wind speed } \\
(\%)\end{array}$ & $\begin{array}{c}\text { ETo } \\
(\mathrm{mm})\end{array}$ & $\begin{array}{c}\text { Precipitation } \\
(\mathrm{mm})\end{array}$ \\
\hline Dec & 25,3 & 66 & 1,6 & 3,0 & 5,0 \\
Jan & 23,5 & 77 & 0,9 & 1,8 & 384,5 \\
Feb & 22,8 & 76 & 0,7 & 2,6 & 179,3 \\
Mar & 23,0 & 82 & 0,8 & 2,3 & 241,3 \\
Apr & 23,0 & 84 & 1,0 & 2,0 & 116,5 \\
\hline
\end{tabular}


Tabela 3. Coeficientes de cultivo $(\mathrm{Kc})$ da helicônia nas diferentes fases da cultura, a partir da evapotranspiração de referência estimada pelo tanque Classe A, e pelo método de Penman-Monteith-FAO.

Table 3. Heliconia crop coefficients (Kc) for different development stages, estimated from reference evapotranspiration for both Class A pan evaporation and Penman-Monteith-FAO equation.

\begin{tabular}{clcc}
\hline Daye after planting & \multicolumn{1}{c}{ Deve lopment stages } & Kc Classe A pan & Kc Penman-Monteith-FAO \\
\hline $01-45$ & F1 ( from initial to 10\% canopi cover) & - & - \\
$60-154$ & F2 (10\% to 80\% canopi cover) & 0,80 & 0,60 \\
154172 & F3 (80\% crop canopi to blooming beginng) & 1,20 & 0,80 \\
$175-185$ & F4 ( from blooming to harvest start) & 1,20 & 0,80 \\
\hline
\end{tabular}

